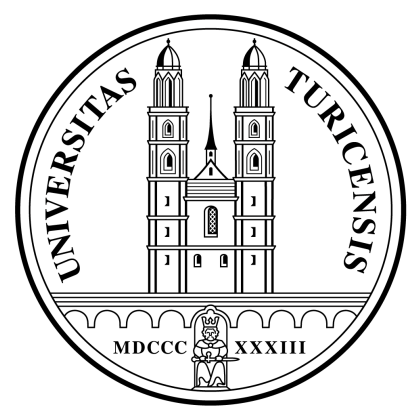

Institute for Empirical Research in Economics

University of Zurich

Working Paper Series

ISSN 1424-0459

Working Paper No. 71

An Extension of Mantel (1976) to Incomplete Markets

Thorsten Hens

January 2001 


\title{
An Extension of Mantel (1976) to Incomplete Markets
}

\author{
Thorsten Hens \\ Institute for Empirical Research in Economics \\ University of Zurich \\ Blümlisalpstrasse 10 \\ CH-8006 Zurich \\ Switzerland
}

January 23, 2001

\begin{abstract}
In the incomplete markets model with numeraire asset and a single consumption good we show that, even with homothetic preferences, on compact sets of prices Continuity, Walras' identity and Homogeneity characterize the properties of market excess demand. This result is proved by an extension of Mantel (1976) to the case of incomplete markets.

Journal of Economic Literature Class. No. : D 52, C 62, G 10.
\end{abstract}




\section{Introduction}

In this note we show that under some conditions, even when markets are incomplete, on compact sets of prices Continuity, Walras' Law and Homogeneity characterize the properties of market excess demand.

For the complete markets model this statement has been shown to be true in a series of papers starting from Sonnenschein (1973a), (1973b), Debreu (1974) and Mantel (1974),(1976). For the incomplete markets model, recently similar results have been found. Bottazzi and Hens (1996), Gottardi and Hens (1998), Gottardi and Mas-Colell (1999) and Chiappori and Ekeland (1999) demonstrate that by restricting attention to a point or to small open neighborhoods of regular prices these results carry over to the case of real assets, the case of nominal assets and to the case of demand being defined on the Grassmanian, respectively.

The result presented in this note holds for arbitrary compact sets of prices, however we restrict attention to the incomplete markets model with real assets and a single consumption good in each state. This case is of particular importance for financial economics. Whereas Debreu's (1974) proof cannot be applied to the incomplete markets setting in order to obtain a global decomposition result, as we demonstrate in the appendix, we show that Mantel's (1976) proof based on duality theory nicely carries over to the setting of this note. Indeed, following Mantel's (1976) approach one can generate any excess demand even with homothetic preferences. The case of homothetic preferences is of particular importance for aggregation results because in this case demand has a lot of structure. If utility functions were identical or if endowments were colinear then, in the case of homothetic preferences, market demand is not arbitrary but can be generated by a representative consumer. For the incomplete markets model aggregation in the case of homothetic and identical preferences has been shown by Hens (1990) and by Detemple and Gottardi (1998), generalizing the theorems of Antonelli (1886) and of Gorman (1953), respectively. The case of homothetic preferences and colinear endowments has been proved for the incomplete markets model by Voß (1997), who generalizes a result of Chipman (1974).

\section{Mantel's Theorem}

In order to put our result into the correct perspective we will briefly recall Mantel's Theorem.

Let $\mathbb{R}^{n}$ be the commodity space and let $H:=\left\{p \in \mathbb{R}^{n} \mid \sum_{l=1}^{n} p_{l}=1\right\}$ be the set of normalized prices. For every compact convex subset $P \subset H$ Mantel defines $P^{*}:=\left\{x \in \mathbb{R}^{n} \mid p \cdot x \geq 0\right.$ for all $\left.p \in P\right\}$ the positive polar of the set $P$. Given these definitions we can state 
Theorem (Mantel (1976))

Let $P \subset H$ be compact, convex. Let $Z: P \rightarrow \mathbb{R}^{n}$ be $C^{2}$ on $P$ and satisfy $p \cdot Z(p)=0$ for all $p \in P$. Let $\omega^{i} \in P^{*}, i=1, \ldots, n$ be independent vectors. Then there exists a real $k>0$, a convex cone $X \subset P^{*}$, and $n$ unsatiated consumers with strictly concave, homogeneous utility functions $U^{i}: X \rightarrow \mathbb{R}$ and initial endowments $k \omega^{i}$, whose excess demand functions add up to $Z$ on $P$.

If one wants to apply Mantel's Theorem to the standard Arrow-Debreu model (see e.g. Theorem 7 in Shafer and Sonnenschein (1982)) one could set $P=\left\{p \in \mathbb{R}^{n} \mid p_{l} \geq \epsilon, l=1, \ldots, n \quad \sum_{l=1}^{n} p_{l}=1\right\}$ and gets that $P^{*}$ comes close to the nonnegative orthant for small $\epsilon>0$.

\section{The Model}

In the following, we apply Mantel's theorem to the market excess demand for assets in an incomplete markets model with a single consumption good, numeraire assets and two periods. To this end let there be a single consumption good available in the second period. There is symmetric uncertainty because in the second period one of $s=1, \ldots, S$ states realizes. Consumers $i=1, \ldots, I$ are endowed with state contingent resources $\omega^{i} \in \mathbb{R}_{+}^{S}$ and they evaluate state contingent consumption $x^{i}$ according to continuous, monotone and strictly quasi-concave utility functions $U^{i}: \mathbb{R}_{++}^{S} \mapsto \mathbb{R}$. To insure against the uncertainty, in the first period consumers can buy and sell without any short sales restrictions $j=1, \ldots, J$ assets which are described by their state contingent payoffs $A_{s j}, j=1, \ldots, J, s=1, \ldots, S$. On denoting by $q$ the vector of asset prices and by $A \in \mathbb{R}^{S \times J}$ the matrix of asset payoffs, the consumer's decision problem can therefore be written as:

$$
\bar{g}^{i}(q):=\arg \max _{\theta \in \Theta} U^{i}\left(\omega^{i}+A \theta\right) \quad \text { s.t. } \quad q \cdot \theta \leq 0,
$$

where $\bar{g}^{i}$ denotes the consumer's (excess) demand function for assets. The market excess demand for assets is correspondingly defined as $G(q):=\sum_{i} \bar{g}^{i}(q)$. Note that excess demand is only well defined if asset prices do not allow for arbitrage, i.e. if $q$ is in the set

$$
\begin{gathered}
Q:=\left\{q \in R^{J} \mid \text { there is no } \theta \text { with }\left(\begin{array}{c}
-q^{T} \\
A
\end{array}\right) \theta>0\right\} \\
=\left\{q \in \mathbb{R}^{J} \mid q=A^{T} \pi \text { for some } \pi \in \mathbb{R}_{++}^{S}\right\}
\end{gathered}
$$


Furthermore, note that in this model, it may happen that all asset prices are negative, so that the price normalization chosen by Mantel is not appropriate. Since asset prices must be no-arbitrage prices, using the existence of strictly positive state prices, we will normalize the set of asset prices indirectly by a normalization on state prices $\pi$ in the standard Walrasian way, i.e. $Q_{\epsilon}:=\left\{q \in \mathbb{R}^{J} \mid q=A^{T} \pi\right.$ for some $\left.\pi \in S_{\epsilon}\right\}$, where $S_{\epsilon}:=\left\{\pi \in \mathbb{R}_{++}^{S} \mid \pi_{s} \geq \epsilon, s=1, \ldots, S\|\pi\|=1\right\}$. In our main result the set $X:=\left\{x \in \mathbb{R}_{++}^{S} \mid x=A \theta\right.$ for some $\left.\theta \in \mathbb{R}^{J}\right\}$ and the preimage of $X$ under the linear mapping $A$, the set $\Theta:=\left\{\theta \in \mathbb{R}^{J} \mid A \theta \gg 0\right\}$, will be of considerable interest. We will choose $\omega^{i} \in X$ so that the GEI economy, which we construct to generate the asset market excess demand, will in particular satisfy the spanning condition $\omega^{i} \in\langle A\rangle, i=1, \ldots, I$. Moreover by an application of a result by Chipman, Hurwicz and Uzawa (see Lemma 1 of this note) we get the stronger conclusion that the utility functions (defined on subsets of $X$ ) are not only unsatiated but monotone.

\section{Main Result}

The following proposition will be shown to be true.

Main Result Let $A \in \mathbb{R}^{S \times J}$ be any asset structure with rank $A=J$ and $A \theta>0$ for some $\theta \in \mathbb{R}^{J}$. Furthermore, let $G: Q \rightarrow \mathbb{R}^{J}$ be $C^{1}$ and $C^{2}$ on $Q_{\epsilon}$ and let $G$ satisfy $G(\lambda q)=G(q)$ for all $q \in Q, \lambda>0$, and $q \cdot G(q)=0$ for all $q \in Q$. Then for every $\epsilon>0$ the following is true: Let $\omega^{i} \in X, i=1, \ldots, J$ be linearly independent vectors, then there exists a real $k>0$, and $J$ consumers with continuous strictly quasi-concave, monotone, linearly homogeneous utility functions $U^{i}: X \rightarrow \mathbb{R}$ and initial endowments $k \omega^{i}$, whose asset excess demand functions add up to $G$ on $Q_{\epsilon}$.

To prove this theorem we do not directly use duality theory but follow a hint given by Richter (which was made public in Shafer and Sonnenschein (1982)) and write down the demand functions directly in order to apply the Integrability Theorem for demand functions which are linear in income.

Let $\Omega_{\epsilon}:=\left\{\pi \in \mathbb{R}_{++}^{S} \mid \pi_{s}>\epsilon, s=1, \ldots, S\right\} \times \mathbb{R}_{+}$for all $\epsilon \geq 0$ and let $X_{f}$ be the image of a function $f$ defined on $\Omega_{\epsilon}$, i.e. $X_{f}=f\left(\Omega_{\epsilon}\right)$. Endowed with this notation we recall from Chipman (1974) and Hurwicz and Uzawa(1971):

Lemma (Chipman (1974) and Hurwicz and Uzawa(1971))

Let $f$ satisfy the following six properties: 
(A) $f: \Omega_{\epsilon} \rightarrow \mathbb{R}_{+}^{S}$,

(B) $\pi \cdot f(\pi, b)=b$

(D) $f$ is differentiable

for all $(\pi, b) \in \Omega_{\epsilon}$,

(L) $f(\pi, \lambda b)=\lambda f(\pi, b)$

on $\Omega_{\epsilon}$,

(S) $\partial_{\pi} f(\pi, b)$ is symmetric

for all $(\pi, b) \in \Omega_{\epsilon}$ all $\lambda \geq 0$,

$(N) \partial_{\pi} f(\pi, b)$ is negative semi-definite

Then there exists a continuous, strictly quasi-concave, monotone, linearly homogeneous function $U: X_{f} \rightarrow \mathbb{R}$ such that $f(\pi, b)=\arg \max _{x \in X_{f}} U(x)$,

s.t. $\pi \cdot x \leq b$.

\section{A Technical Remark}

The lemma stated above cannot be found in exactly the way we stated it. One difference is that Chipman, Hurwicz and Uzawa let the function $f$ be defined on $\Omega_{\epsilon}$ for $\epsilon=0$. But for any other $\epsilon \geq 0$ the theorem is still true, it only will become progressively weaker if $\epsilon$ is larger. This can be seen from a careful reading of the proof given in Hurwicz and Uzawa (1971). The key mathematical step is to solve a system of partial differential equations, which can be done for every $\epsilon \geq 0$, as long as the Frobenius integrability conditions are satisfied. These conditions applied to the function $f$ require the Slutzky matrix of $f, S_{f}(\pi, b):=\partial_{\pi} f(\pi, b)+\partial_{b} f(\pi, b) f^{T}(\pi, b)$ to be negative semi-definite and symmetric. Another difference is that instead of requiring these properties of the Slutzky matrix, we assure these properties by the analogous ones of the Jacobian matrix; conditions $(\mathrm{S})$ and $(\mathrm{N})$. This is correct, because it is well known that negative semi-definiteness of the Jacobian $\partial_{\pi} f(\pi, b)$ implies negative semi-definiteness of the Slutzky matrix $S_{f}(\pi, b)$ see e.g. Hildenbrand and Jerison (1988). Finally, with linear Engelcurves (i.e. assumption (L)) we get $\partial_{b} f(\pi, b) f^{T}(\pi, b)=\frac{1}{b} f(\pi, b) f^{T}(\pi, b)$ so that symmetry carries over as well.

In the proof of our main result we will use the definition of excess demand as excess demand for assets and that as excess demand for income transfers. The first description is easier when one wants to guarantee that excess demand stays in $\langle A\rangle$. The second definition is more convenient when one wants to apply results (like the theorem of Chipman, Hurwicz and Uzawa) that have been proved in the Arrow-Debreu model. Since it is immediate how to transfer one excess demand approach into the other we will go back and forth between the two approaches whenever this allows to shorten the proof. We have stated our main result in terms of asset excess demand. However, as will be seen from our proof, which uses both formulations of excess demand, we have then also shown the corresponding result for the income excess demand. 


\section{Proof (of main result)}

We first follow the decomposition given by Mantel (1976). Let $\bar{\theta}^{i} \in \Theta$ be the unique $\theta \in \mathbb{R}^{J}$ such that $\omega^{i}=A \bar{\theta}^{i}, i=1, \ldots, J$. Take any $J$ independent vectors $c^{i} \in H:=\left\{y \in \mathbb{R}_{++}^{J} \mid \sum_{j=1}^{J} y_{j}=1\right\}$. Define the $J \times J$ matrices $W=\left(\bar{\theta}^{1}, \ldots, \bar{\theta}^{J}\right) ; C=\left(c^{1}, \ldots, c^{J}\right) ; B=C W^{T} ;$ and $M=\left(W^{T}\right)^{-1}$.

Before we proceed, note that $\forall q \in Q, B q=C W^{T} q \gg 0$ since $q^{T} \bar{\theta}^{i}=$ $\pi^{T} A \bar{\theta}^{i}=\pi^{T} \omega^{i} \geq 0$ and $W^{T} q \neq 0$.

$$
\text { Let } g^{i}\left(q, b^{i}\right):=\underbrace{\left[-\left(\frac{1}{k}\right) \partial_{q} G^{T}(q) m^{i}+B^{T} \widehat{B q}^{-1} c^{i}\right]}_{\tilde{g}^{i}(q)} b^{i}
$$

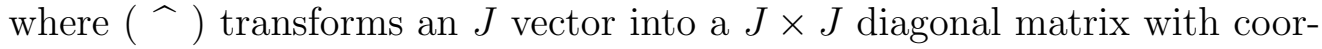
dinates of the vector along its main diagonal and where $m^{i}$ is the ith column of M.

Define by $\mathbb{I}$ the column vector $(1, \ldots, 1)^{\top}$ and consider the aggregate asset demand:

$$
\begin{aligned}
\sum_{i} g^{i}\left(q, q \cdot k \bar{\theta}^{i}\right) & =-\partial_{q} G^{T}(q) M W^{T} q+k B^{T} \widehat{B q}^{-1} C W^{T} q \\
& =-\partial_{q} G^{T}(q) q+k B^{T} \widehat{B q}^{-1} B q \\
& =G(q)+k B^{T} \mathbb{I} \quad \text { (from Walras Law) } \\
& =G(q)+k W C^{T} \mathbb{I} \quad \\
& =G(q)+k \sum_{i} \bar{\theta}^{i}
\end{aligned}
$$

Thus the $g^{i}\left(q, q k \bar{\theta}^{i}\right)-k \bar{\theta}^{i}$ add up to $G(q)$.

So far we are in line with Mantel's proof. But now we deviate from it and show that for every $\epsilon>0$ there exists some $k>0$ such that the asset demand functions $g^{i}\left(q, b^{i}\right), i=1, \ldots, I$ satisfy the following six properties:
(更) $A g^{i}\left(q, b^{i}\right) \in \mathbb{R}_{+}^{S}$
(苂) $q \cdot g^{i}\left(q, b^{i}\right)=b^{i}$
$\left(q, b^{i}\right) \in \tilde{Q}_{\epsilon}$
$(\tilde{D}) g^{i}$ is differentiable
for all $\left(q, b^{i}\right) \in \tilde{Q}$
$(\tilde{L}) g^{i}\left(q, \lambda b^{i}\right)=\lambda g^{i}\left(q, b^{i}\right)$
on $\tilde{Q}_{\epsilon}$
for all $\left(q, b^{i}\right) \in \tilde{Q}$ 
$(\tilde{S}) \partial_{q} g^{i}\left(q, b^{i}\right)$ symmetric $\quad$ for all $\left(q, b^{i}\right) \in \tilde{Q}$

$(\tilde{N}) \partial_{q} g^{i}\left(q, b^{i}\right)$ negative semi-definite for all $\left(q, b^{i}\right) \in \tilde{Q}_{\epsilon}$

where $\tilde{Q}_{\epsilon}:=Q_{\epsilon} \times \mathbb{R}_{+}$and $\tilde{Q}:=Q \times \mathbb{R}_{+}$.

The arguments are as follows:

$(\tilde{D})(\tilde{L})$ are given by assumption.

$(\tilde{A})$ : It is sufficient to show that $A B^{T} y \gg 0$ for all $y \in \mathbb{R}_{++}^{J}$, because for $k>0$ large enough the second term in (1) dominates the first and because $Q_{\epsilon}$ is compact. But $A B^{T} y=A W C^{T} y \gg 0$ since $\bar{\theta}^{i} \in \Theta$.

$(\tilde{B})$ :

$$
\begin{array}{r}
q \cdot g^{i}\left(q, b^{i}\right)=q \cdot\left[-\left(\frac{1}{k}\right) \partial_{q} G(q)^{T} m^{i}+B^{T} \widehat{B q}^{-1} c^{i}\right] b^{i} \\
=\left[-\left(\frac{1}{k}\right) q \partial_{q} G^{T}(q) m^{i}+q B^{T} \widehat{B q}^{-1} c^{i}\right] b^{i}=\left[1 c^{i}\right] b^{i}=b^{i}
\end{array}
$$

In the last equation $q^{T} \partial_{q} G^{T}(q)=0$ follows from differentiating $G(\lambda q)=G(q)$ with respect to $\lambda$.

To verify $(\tilde{N})$ and $(\tilde{S})$ we note that $\tilde{g}^{i}(q)$ is the gradient of $v^{i}(q):=$ $-\left(\frac{1}{k}\right) m^{i} G(q)+c^{i} \log B q$. Thus the second derivative of $v^{i}$ is symmetric and since $G$ is $C^{2}$, there exists $k>0$ large enough so that $v^{i}(q)$ is concave on the compact set $Q_{\epsilon}$.

In order to apply Lemma 1 we transfer the asset demand problem to its dual, the income transfer problem. I.e. we consider $f^{i}: \Omega \rightarrow X$ defined by

$$
f^{i}\left(\pi, b^{i}\right):=A g^{i}\left(A^{T} \pi, b^{i}\right)
$$

and verify that $f^{i}$ satisfies $(\mathrm{A}),(\mathrm{B}),(\mathrm{D}),(\mathrm{L}),(\mathrm{S}),(\mathrm{N})$.

(A), (B), (L), (D), follow directly from $(\tilde{A}),(\tilde{B}),(\tilde{D}),(\tilde{L})$ respectively.

To verify (S) (N) we fix $b^{i}$ and differentiate (2) with respect to $\pi$

$$
\partial_{\pi} f^{i}\left(\pi, b^{i}\right)=A \partial_{q} g^{i}\left(q, b^{i}\right) A^{T} .
$$

¿From (3) we see that the Jacobian of income excess demand inherits the negative semi-definiteness and symmetry from the Jacobian of asset excess 
demand.

Thus we are in a position to apply the lemma and get that

$$
f^{i}\left(\pi, b^{i}\right)=\arg \max _{x \in X_{f^{i}}} U^{i}(x) \quad \pi \cdot x \leq b^{i},
$$

where $X_{f^{i}} \subset\langle A\rangle \cap \mathbb{R}_{+}^{S}$ and $U^{i}$ is continuous, monotone, strictly quasi-concave and linearly homogeneous.

Resubstitution of $A \theta$ for $x$ and $q$ for $A^{T} \pi$ leads to

$$
g^{i}\left(q, q \cdot k \bar{\theta}^{i}\right)=\arg \max _{\theta \in \Theta} U^{i}(A \theta) \quad q \theta \leq q k \bar{\theta}^{i}
$$

i.e. $g^{i}$ is the asset demand function derived from a continuous, monotone and strictly quasi concave utility function, which is linearly homogeneous.

\section{A Final Remark}

Having shown that Mantel's theorem can be generalized to incomplete markets in the case of numeraire assets and a single consumption good, one might wonder whether it also carries over to the general incomplete markets model. However, with general assets its is well known that excess demand in incomplete markets is not continuous which hinders straightforward applications of integrability results like that of Chipman, Hurwicz and Uzawa because these are based on the continuity of the demand function. In the case of numeraire assets it is also not immediate how to generalize Mantel's theorem when there are multiple commodities in each state. Using the definition of excess demand as being excess demand for asset and spot-markets (cf. Magill and Shafer (1991), Definition 3) it is not immediate how duality theory (or integrability theory) can be applied because in this case excess demand is derived from several budget constraints. And following the dual no-arbitrage description (cf. Magill and Shafer (1991), Definition 4) (which is the generalization of the above mentioned income excess demand) the problem then lies in the way Mantel decomposes excess demand. The seminal idea of Mantel is to decompose excess demand into the sum of a well behaved term which one is free to choose (independently from the given excess demand), and a term being determined by the given market excess demand (compare equation (1) of our paper or equation (2) of Mantel (1976)). By choosing the size of the endowments Mantel then assures that the sum of both terms can be controlled by the well behaved part. Here controlled means that the negative definiteness of the Jacobian of the well behaved term will eventually guarantee negative definiteness of the sum of both terms. In incomplete 
markets, using this decomposition for the no-arbitrage approach, the sum of both terms will have to satisfy the spanning constraint. However, in order to get spanning changing the relative size of the two terms does not help.

\section{Appendix}

The theorem we have proved above is the first global decomposition result of excess demand when markets are incomplete. Moreover it is the first decomposition result in incomplete markets that holds even with homothetic preferences. Our result was obtained from a careful application of the seminal result of Mantel (1976). The degree to which we could borrow arguments from Mantel (1976) was surprising to us because, as we show now, without further restrictions, the competing decomposition result of Debreu (1974) is not applicable to obtain a global decomposition result when markets are incomplete.

To this end let $p \in \mathbb{R}^{n} \backslash\{0\}$ be some non-zero price vector and let $z(p) \in$ $L(p) \subset p^{\perp}$ be the excess demand at $p$ which lies in some linear subspace being a subset of the space orthogonal to $p$.

In this abstract formulation Debreu's (1974) decomposition goes as follows: Add to $z(p)$ some positive multiple, say $\lambda$, of the price vector $p$ and decompose the resulting vector $z(p)+\lambda p$ into the weighted sum of the $n$ unit vectors in $\mathbb{R}^{n}$, where the weights are the $n$ coordinates of the vector $z(p)+\lambda p$. Finally apply the orthogonal projection onto $L(p)$, say $\operatorname{proj}_{L(p)}$, to the resulting equation to obtain the decomposition:

$$
z(p)=\sum_{i=1}^{n} \beta_{i}(p) \operatorname{proj}_{L(p)} e_{i} .
$$

where $e_{i}$ denotes the $\mathrm{i}$-th unit vector in $\mathbb{R}^{n}$.

In the rationalization step of Debreu's (1974) proof the residual vectors

$$
\nabla_{i}=e_{i}-\operatorname{proj}_{L(p)} e_{i}
$$

are of considerable importance because by construction of the preferences they will become the gradient vectors of the utility functions.

This general procedure can be applied to the asset excess demand and to the income excess demand of the incomplete markets model. In both cases, as we show next, the monotonicity of preferences cannot be guaranteed. 


\section{Asset excess demand}

In this case the linear subspace $L(p)$ is the space of vectors orthogonal to the asset price vector, i.e. the space $q^{\perp}$. Hence applying Debreu's decomposition, the gradient of the indirect utility of assets, $V^{i}\left(\theta^{i}\right)=U^{i}\left(\omega^{i}+A \theta^{i}\right)$, which we denote by $\nabla^{i}$, is colinear to the asset price vector $q$, say $\nabla^{i}=\alpha^{i} q$. Monotonicity of the direct utility function $U^{i}\left(x^{i}\right)$ requires that $A \nabla^{i} \in \mathbb{R}_{++}^{S}$. By the no-arbitrage relation this is equivalent to $\alpha^{i} A A^{\top} \pi \in \mathbb{R}_{++}^{S}$. This need however not be the case. For example choose an asset matrix with two assets and two states such that the scalar product of the two different rows of $A$ is negative. Then $A A^{\top}$ is a two dimensional square matrix with negative off diagonal. Thus some $\pi \in \mathbb{R}_{++}^{2}$ can be found for which $\alpha^{i} A A^{\top} \pi$ has a positive and a negative component. Hence the income utility function is not monotone.

\section{Income excess demand}

Let $n=3, p=c \mathbb{I}, A=(1,1,-2)^{\top}$ and let $L(p)=p^{\perp} \cap\langle A\rangle$, where $c=1 / \sqrt{3}$. Then

$$
\operatorname{proj}_{L(p)} e_{1}=A\left(A^{\top} A\right)^{-1} A^{\top} e_{1}=(1 / 6)(1,1,-2)^{\top}
$$

and

$$
\nabla_{1}=e_{1}-\operatorname{proj}_{L(p)} e_{1}=(1 / 6)(5,-1,2)^{\top} \notin \mathbb{R}_{++}^{3}
$$

is the gradient vector of the direct utility function $U^{1}\left(\omega^{1}+z^{1}\right)$, which is not monotone either.

Because of the monotonicity problem that arises in applying Debreu's (1974) proof to incomplete markets Bottazzi and Hens (1996) have chosen a different decomposition which is however not able to generate excess demand globally. 


\section{References}

Antonelli (1886) : Sulla Teoria Matematica della Economia Politica, Pisa; Nella Tipografia del Folchetto. English translation by J.S. Chipman and A.P. Kirman, chapter 16 in J.S. Chipman,L. Hurwicz, M.K. Richter and H.F. Sonnenschein (eds.): Preferences, utility and demand,New York: Harcourt Brace Jovanovich, Inc. 1971, 333-360.

Bottazzi, J.M. and Hens, Th. (1996) : Excess demand functions and incomplete markets, Journal of Economic Theory 68, 49-63.

Chiappori, P.A. and Ekeland, I. (1999) : Disaggregation of excess demand function in incomplete markets, Journal of Mathematical Economics 31, 111-129.

Chipman, J.S. (1974) : Homothetic preferences and aggregation, Journal of Economic Theory 8, 26-38.

Debreu, G. (1974) : Excess demand functions, Journal of Mathematical Economics 1, 15-23.

Detemple, J. and P. Gottardi (1998) : Aggregation, Efficiency and Mutual Fund Separation,Economic Theory 11, 443-455.

Eisenberg, B. (1961) : Aggregation of utility functions, Management Science $7,337-350$.

Gorman, W.M. (1953) : Community indifference curves, Econometrica, $21,63-80$.

Gottardi P. and Hens, Th. (1999) : Disaggregation of excess demand and comparative statics with incomplete markets and nominal assets, Economic Theory 13, 287-308.

Hildenbrand, W. and Jerison, M. (1989) : The demand theory of the weak axiom of revealed preference, Economic Letters 29, 209-213.

Hurwicz, L. and H. Uzawa (1971) : On the integrability of demand functions, chapter 6 in Chipman, J.S., L. Hurwicz, M.K. Richter and H.F. Sonnenschein, eds., Preferences, utility and demand. New York: Harcourt Brace Jovanovich, Inc., , 114-148.

Magill, M. and W. Shafer (1991) : Incomplete Markets; Chapter 30, in Handbook of Mathematical Economics, vol. IV, W. Hildenbrand and H. F. Sonnenschein (eds.), New York: North-Holland, 71-95. 
Mantel, R. (1976) : Homothetic preferences and community excess demand functions, Journal of Economic Theory 12, 197-201.

Mantel, R. (1974) : On the characterization of aggregate excess demand, Journal of Economic Theory 7, 348-353.

Shafer, W. and H.F. Sonnenschein (1982) : Market demand and excess demand functions; Chapter 14, in Handbook of Mathematical Economics, vol. II, K. Arrow and M. Intrilligator (eds.), New York: NorthHolland , 71-95.

Sonnenschein, H.F. (1973) : Do Walras' identity and continuity characterize the class of community excess demand functions?, Journal of Economic Theory 6, 345-354.

Sonnenschein, H.F. (1973) : The utility hypothesis and market demand theory, Western Economic Journal 11, 404-410.

Voß, B. (1997) : Eindeutigkeit von Gleichgewichten bei unvollstndigen Finanzmärkten, Diploma Thesis, University of Bielefeld. 